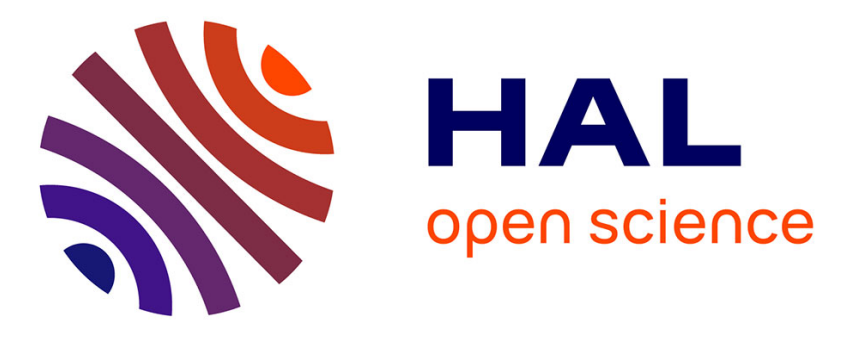

\title{
L'anxiété préopératoire : manifestations cliniques, évaluation et prévention
}

R. Amouroux, C. Rousseau-Salvador, D. Annequin

\section{To cite this version:}

R. Amouroux, C. Rousseau-Salvador, D. Annequin. L'anxiété préopératoire : manifestations cliniques, évaluation et prévention. Annales Médico-Psychologiques, Revue Psychiatrique, 2010, 168 (8), pp.588. 10.1016/j.amp.2009.10.017 . hal-00682246

\section{HAL Id: hal-00682246 \\ https://hal.science/hal-00682246}

Submitted on 24 Mar 2012

HAL is a multi-disciplinary open access archive for the deposit and dissemination of scientific research documents, whether they are published or not. The documents may come from teaching and research institutions in France or abroad, or from public or private research centers.
L'archive ouverte pluridisciplinaire HAL, est destinée au dépôt et à la diffusion de documents scientifiques de niveau recherche, publiés ou non, émanant des établissements d'enseignement et de recherche français ou étrangers, des laboratoires publics ou privés. 


\section{Accepted Manuscript}

Title: L'anxiété préopératoire: manifestations cliniques, évaluation et prévention

Authors: R. Amouroux, C. Rousseau-Salvador, D. Annequin

PII: $\quad$ S0003-4487(10)00223-4

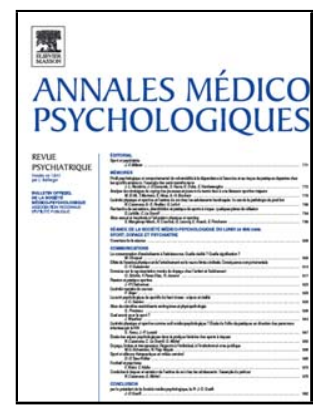

DOI: $\quad$ doi:10.1016/j.amp.2009.10.017

Reference: $\quad$ AMEPSY 1197

To appear in: $\quad$ Annales Médico-Psychologiques

Received date: 26-3-2009

Accepted date: $\quad 8-10-2009$

Please cite this article as: Amouroux R, Rousseau-Salvador C, Annequin D, L'anxiété préopératoire: manifestations cliniques, évaluation et prévention, Annales mediopsychologiques (2010), doi:10.1016/j.amp.2009.10.017

This is a PDF file of an unedited manuscript that has been accepted for publication. As a service to our customers we are providing this early version of the manuscript. The manuscript will undergo copyediting, typesetting, and review of the resulting proof before it is published in its final form. Please note that during the production process errors may be discovered which could affect the content, and all legal disclaimers that apply to the journal pertain. 


\title{
Mémoire
}

L'anxiété préopératoire : manifestations cliniques, évaluation et prévention

Preoperative anxiety: clinical manifestations, evaluation and prevention

R. Amouroux *, C. Rousseau-Salvador, D. Annequin

Unité Fonctionnelle d'Analgésie Pédiatrique, Hôpital Armand Trousseau, 26, avenue du Docteur Arnold Netter, 75571 Paris cedex 12, France

Auteur correspondant: R. Amouroux, Unité Fonctionnelle d'Analgésie Pédiatrique, Hôpital Armand Trousseau, 26, avenue du Docteur Arnold Netter, 75571 Paris cedex 12, France Tél. : 0144736519

Fax : 0149280211

Adresse email : remy.amouroux @ trs.aphp.fr

Texte reçu le 26 mars 2009, accepté le 8 octobre 2009

\section{Résumé}

L'anxiété préopératoire est un malaise physique et psychologique dont l'apparition est consécutive à la possibilité d'une intervention chirurgicale. Lorsqu'elle est d'intensité faible à modérée, c'est une réponse adaptée à cette situation potentiellement anxiogène. Il a été établi que des niveaux importants d'anxiété préopératoire augmentent le risque de complications postopératoires, ainsi que la survenue de troubles émotionnels et comportementaux.

Malgré ces conséquences médicales et psychologiques parfois graves, il n'y a que très peu de littérature destinée aux professionnels de santé francophones sur le sujet. L'objectif de cet article est d'effectuer une synthèse de la notion d'anxiété préopératoire, de ses manifestations cliniques, ainsi que de ses moyens d'évaluation et de prévention.

Mots clés : Anesthésie ; Anxiété ; Chirurgie ; Périopératoire

\begin{abstract}
Preoperative anxiety consists of physiological and psychological discomfort that manifests itself at the possibility of a surgical intervention. When the anxiety is weak or moderate it is considered to be an adaptive response to a potentially anxiety producing situation. Prior research has established that high levels of preoperative anxiety increase the
\end{abstract}


risk for postoperative complications as well as emotional and behavioral difficulties. Despite these sometimes severe medical and psychological consequences, very little literature exists aimed at French health care workers on the subject. The goal of this article is to synthesize the existing knowledge on preoperative anxiety, including its clinical manifestations and methods of evaluation and prevention.

Keywords: Anesthesia; Anxiety; Perioperative; Surgery

\section{Introduction}

L'éventualité d'une intervention chirurgicale suscite différentes réactions qui dépendent notamment de la nature des motifs médicaux, de l'âge et de l'expérience chirurgicale et anesthésique préalable. Le concept d'anxiété préopératoire désigne une forme de malaise physique et psychologique dont les manifestations et l'intensité sont variables, et qui peut avoir un impact important sur les suites médicales et psychologiques de l'intervention. L'objectif de cet article est d'effectuer une synthèse de la notion d'anxiété préopératoire, de ses manifestations cliniques, ainsi que de ses moyens d'évaluation et de prévention.

\section{Le concept et la clinique de l'anxiété préopératoire}

\subsection{Spécificités psychopathologiques de l'anxiété préopératoire}

Il n'est pas aisé de situer précisément l'anxiété préopératoire au niveau psychopathologique, et de la rattacher à une entité nosographique. Si elle est d'intensité faible ou modérée, elle est une réaction adaptée à une situation anxiogène. Lorsqu'elle est massive, elle peut remplir les critères d'une attaque de panique. Chez l'enfant, une expérience chirurgicale précoce négative peut aussi favoriser le développement d'une angoisse de séparation. Si les symptômes perdurent, cela peut mener à l'installation d'une phobie spécifique comme la phobie des soins. Enfin, dans des cas extrêmes et rares, une mauvaise expérience de ce type peut être à l'origine d'un état de stress aigu, voire d'un état de stress post-traumatique. Au-delà de ces aspects nosographiques, il est important de retenir que 
l'anxiété préopératoire n'est pas une catégorie diagnostique. Il s'agit plutôt d'un type d'anxiété spécifique au milieu hospitalier et à l'expérience chirurgicale. C'est cependant différent d'une peur ou d'une phobie de l'anesthésie et/ou de la chirurgie. L'enfant, comme l'adulte, lorsqu'il est confronté à l'expérience de l'hospitalisation dans le cadre d'une intervention chirurgicale, est brusquement plongé dans un monde qu'il ne connaît pas et dont il ne maîtrise pas les règles. C'est cette confrontation à la situation préopératoire qui induit un état d'angoisse. Il ne s'agit donc pas nécessairement de peurs précises ou spécifiques, mais plutôt d'une sensation de malaise, une réaction de stress, qui peut rester confuse ou porter sur différents aspects de l'hospitalisation. Dans cette perspective, l'anxiété préopératoire pourrait être la conséquence d'un débordement de la capacité d'adaptation face au stress généré par une intervention chirurgicale. Certaines attitudes et certains modes de raisonnements, comme le catastrophisme (tendance à la rumination, la résignation, et à la généralisation), pourraient majorer l'apparition de troubles émotionnels et comportementaux.

\subsection{La clinique de l'anxiété préopératoire chez l'enfant}

En pédiatrie, la prévalence de l'anxiété préopératoire varierait entre $40 \%$ et $60 \%$ selon les études [24,36]. Lors d'une hospitalisation, l'enfant est subitement confronté à une situation extraordinaire et inquiétante où la plupart de ses repères sont bousculés. Des psychologues et des psychanalystes pionniers de ce domaine, comme Anna Freud et Thési Bergman, ont ainsi souligné que toute intervention chirurgicale annoncée à l'avance à l'adulte ou à l'enfant peut susciter chez le malade l'attente justifiée et consciente de la douleur, de malaises, de pertes et souvent de mutilations [6]. Joyce Robertson a par ailleurs décrit au jour le jour les modifications subtiles du comportement qu'elle observe chez son enfant de quatre ans avant qu'il ne subisse une amygdalectomie [32]. La spécificité de l'expérience de l'hospitalisation chez l'enfant tiendrait au fait que sa maturité affective étant incomplète, il est confronté à une situation étrange dont il ne comprend pas les règles de fonctionnement. L'hospitalisation et la chirurgie comportent en effet beaucoup de mauvaises surprises potentielles: l'enfant est séparé de ses proches, il va devoir se déshabiller devant des étrangers, il risque d'avoir mal, et il gardera souvent des cicatrices, qu'elles soient morales ou physiques. Dans le cas de nombreuses interventions chirurgicales de routine (végétations, pose d'aérateurs trans-tympaniques, ou encore amygdalectomie), l'enfant sort de l'hôpital dans un état qui peut lui sembler bien pire que celui dans lequel il était à son arrivée. On peut 
aisément comprendre qu'il se méfiera la prochaine fois qu'il sera confronté à des blouses blanches qui lui assurent qu'elles vont le « soigner ». C'est en effet la pensée magique plus que l'évaluation rationnelle des faits qui prédomine chez le jeune enfant. Cela implique notamment qu'en l'absence d'explication satisfaisante, l'enfant interprète les faits de façon erronée. Les maladies peuvent ainsi parfois être comprises comme des punitions qui viennent sanctionner un «mauvais » comportement ou une «mauvaise » pensée [4,6,34].

McGraw a proposé une interprétation développementale des facteurs psychologiques prédisposant à l'anxiété dans un contexte chirurgical [23]. Selon lui, le nouveau-né pourrait dans la majorité des cas être rassuré par l'équipe soignante, et ne ressentirait généralement pas difficilement la séparation avec les parents. À l'inverse, de un à trois ans, la prégnance des liens entre l'enfant et ses parents impliquerait un risque important de développement d'une anxiété de séparation. Les enfants sont alors généralement trop jeunes pour bénéficier d'explications, mais répondent bien à la distraction et à la réassurance. De quatre à six ans, les enfants veulent plus d'explications. Ceux de sept à douze ans veulent être impliqués dans les prises de décisions les concernant. Il convient cependant de ne pas tout discuter lors d'une situation stressante, mais d'en parler en amont, et en dehors d'un contexte anxiogène. Enfin, les adolescents bénéficieront tout particulièrement du respect de leur intimité et de leur autonomie. Cette interprétation offre l'avantage de pointer l'importance de la prise en compte des capacités de communication de l'enfant. Elle doit cependant être pondérée, notamment pour le nouveau-né qui mérite lui aussi une attention particulière.

L'équipe de Zeev N. Kain de Yale a effectué de nombreux travaux qui ont permis de mieux préciser les conséquences négatives liées à l'anxiété préopératoire chez l'enfant. Il a ainsi été démontré qu'un niveau important d'anxiété préopératoire chez l'enfant était corrélé à des scores de douleurs plus élevés, à une consommation d'antalgiques plus importante, et à trois fois plus de risque d'avoir des scores importants de troubles comportementaux postopératoires $[15,19]$. D'autres travaux ont mis en évidence des facteurs de risque pour le développement d'une anxiété préopératoire. Aucune étude n'a établi que le sexe de l'enfant pouvait jouer un rôle dans le développement de l'anxiété préopératoire [41]. En revanche, plusieurs études ont mis en évidence que les jeunes enfants ont plus de risques de développer ce type d'anxiété que des enfants plus âgés. La période de un à cinq ans semble être une phase critique $[15,41]$. De plus, les mauvaises expériences d'hospitalisations préalables augmentent de façon importante le risque d'installation de troubles anxieux lors de futures hospitalisations. Enfin, il existe de nombreux facteurs psychologiques qui doivent être pris en compte. Les enfants qui, selon une échelle $a d$ hoc, ont un tempérament anxieux, timide ou 
inhibé sont plus anxieux au moment de la séparation d'avec les parents [15,17]. Par ailleurs, les enfants qui ont un style de coping passif (c'est-à-dire les enfants qui ont tendance à avoir recours aux stratégies d'évitement, de retrait, ou à la pensée magique pour faire face au stress de la chirurgie) ont un risque plus important d'anxiété préopératoire [39]. L'anxiété préopératoire de l'enfant est enfin généralement corrélée à celle qu'éprouvent ses parents au moment de l'induction anesthésique $[15,17]$.

\subsection{La clinique de l'anxiété préopératoire chez l'adulte}

Chez l'adulte, la prévalence de l'anxiété préopératoire varierait entre $60 \%$ et $80 \%$ selon les études [21,35]. Le contexte joue probablement un rôle important. Ainsi, l'anxiété serait plus importante en chirurgie esthétique que reconstructive [1]. De la même façon, les patients opérés d'un cancer sont plus anxieux que ceux opérés d'une chirurgie orthopédique. Comme chez l'enfant, les patients adultes qui ont des niveaux importants d'anxiété préopératoire ont un réveil postopératoire plus lent, plus compliqué et plus douloureux [18]. Les principaux facteurs de risques chez l'adulte pour ce type d'anxiété sont le cancer, le tabagisme, les troubles psychiatriques (notamment les troubles anxieux et dépressifs), la douleur préopératoire modérée à intense, les chirurgies de lourdeur intermédiaire et le sexe féminin [3]. Il existe peu d'études qui portent spécifiquement sur l'anxiété préopératoire chez le sujet âgé. Celle d'Oberle et de son équipe a mis en évidence l'existence d'un lien entre l'anxiété postopératoire et la douleur [30]. Ils n'ont cependant pas observé de lien entre l'anxiété préopératoire et la douleur.

Les écrits de Miller sont souvent cités dans la littérature sur l'anxiété préopératoire chez l'adulte [26]. Il s'est notamment intéressé à l'annonce diagnostique de maladies graves. Mais ses travaux peuvent tout aussi bien s'appliquer au contexte médical et hospitalier qu'à l'anxiété préopératoire. Selon Miller, les individus réagissent différemment lorsqu'ils sont confrontés à des situations menaçantes. Face à des événements incontrôlables et imprévisibles, le degré de perception de la menace varie selon les personnes. Il distingue principalement deux types de stratégies de coping :

- la première, le Monitoring, consiste à rechercher de l'information pour diminuer l'incertitude et la détresse générée par l'événement menaçant. Cela va de la simple demande de complément d'information jusqu'à la recherche compulsive de tout élément concernant la situation. Ce profil s'accompagne souvent d'un goût prononcé pour l'auto-observation, et d'une focalisation sur tout ce qui pourrait confirmer la dangerosité de la situation. Dans des 
cas extrêmes, l'individu peut être littéralement débordé par des pensées intrusives qui envahissent tout son champ de conscience ;

- la seconde, le Blunting, consiste à avoir recours à la distraction face à un événement menaçant. Cette stratégie cognitive peut s'apparenter à de la distraction, à de l'intellectualisation, à du déni, ou encore à de la réinterprétation positive des événements. L'individu peut aussi chercher à se distraire par différentes activités, ou par la prise de substances psychoactives.

Miller a développé une échelle qui permet de préciser le style préférentiel de chaque individu. Il s'agit de quatre scénarios catastrophes dans lesquels le patient doit s'immerger mentalement. Il doit préciser comment il réagirait dans chacune des situations. Les réponses qu'il donne déterminent son profil (Monitoring ou Blunting) [27]. À court terme, il semblerait que les stratégies de type Blunting soient plus efficaces. Le Monitoring induirait en effet une détresse émotionnelle plus importante. D’une façon générale, les sujets ayant ce profil seraient plus anxieux et anticiperaient davantage les problèmes. Cependant, à moyen et à long terme, certaines stratégies de Blunting, comme le déni, peuvent conduire à des comportements inadaptés. Une autre étude a montré que les personnes qui composent le mieux avec la menace sont celles qui disposent d'une quantité d'information adaptée à leur profil. En effet, les personnes de type Monitoring réagiront d'autant mieux qu'elles recevront une quantité importante d'information. Celles de type Blunting, à l'inverse, préfèreront des données succinctes [28]. Cela implique de prendre en compte les stratégies d'ajustement des individus, et de personnaliser le message informatif préopératoire.

\section{L'évaluation et la prévention de l'anxiété préopératoire}

Face à un phénomène fréquent et potentiellement pathogène comme l'anxiété préopératoire, on s'attend à disposer de nombreux outils de qualité permettant d'en évaluer, tant chez l'enfant que chez l'adulte, les manifestations cliniques. L'évaluation est en effet la condition sine qua non pour la mise en place de moyens efficaces de prévention. En effet, comment savoir ce qui diminue ou ce qui augmente l'anxiété préopératoire si l'on n'a pas de moyens de mesure suffisamment objectifs de ce phénomène? Les Anglo-Saxons disposent d'outils fiables et souvent très spécifiques destinés à la recherche et à la clinique, qui permettent d'évaluer l'anxiété préopératoire. Mais aucun de ces outils n'a été jusqu'à présent traduit et validé en français. Les différents outils qui sont présentés ici doivent nécessairement 
s'accompagner d'une évaluation plus globale du problème, effectuée par exemple au cours d'un ou de plusieurs entretiens cliniques.

\subsection{Tests et échelles d'évaluation de l'anxiété préopératoire}

Il n'existe pas de test psychologique permettant d'évaluer spécifiquement l'anxiété préopératoire. De nombreuses études utilisent un test « généraliste » de l'anxiété : le State and Trait Anxiety Index (STAI) [38] chez l'adulte, ou le State and Trait Anxiety Index for Children (STAIC) [37] chez l'enfant. Ce test est en effet considéré dans les pays anglo-saxons comme le gold-standard pour l'évaluation de l'anxiété. Cette épreuve psychométrique, dont l'usage est pertinent dans le cadre de la recherche, n'est pas toujours adaptée à une utilisation clinique. Le STAI et le STAIC ont été traduits en français.

On dispose de plusieurs échelles d'hétéro-évaluation de l'anxiété préopératoire. La plus connue et la plus utilisée chez l'enfant est la modified Yale Preoperative Anxiety Scale (mYPAS), qui est une version révisée et étendue de la Yale Preoperative Anxiety Scale (YPAS) [14] (Annexe 1). L'Induction Compliance Checklist (ICC) [16] porte plus spécifiquement sur la compliance de l'enfant lors de l'anesthésie. La Global Mood Scale (GMS) [5] permet d'évaluer l'anxiété concernant les injections chez l'enfant. Elle a aussi été utilisée pour l'anxiété préopératoire.

On dispose aussi d'échelles d'autoévaluation de l'anxiété préopératoire. L'Amsterdam Preoperative Anxiety and Information Scale (APAIS) [25] est un outil réservé à l'adulte, qui combine l'évaluation de l'anxiété à l'évaluation des attentes concernant l'information sur l'intervention (Annexe 2). Elle prend en compte les travaux de Miller sur le Blunting et le Monitoring. Enfin, une échelle d'évaluation de la douleur, la Visual Analogic Scale (VAS), ou Échelle Visuelle Analogique (EVA), peut parfois être utilisée chez l'enfant et chez l'adulte pour évaluer l'anxiété préopératoire. On a montré que le score EVA de l'anxiété était corrélé avec le STAI [20].

\subsection{La prévention de l'anxiété préopératoire}

L'anxiété préopératoire peut favoriser le développement de complications postopératoires diverses, ainsi que de troubles comportementaux et émotionnels. Pour contrecarrer ces méfaits, outre la consultation médicale de pré-anesthésie, des stratégies de prévention ont été élaborées chez l'enfant et l'adulte $[1,43,44]$. En plus de l'approche 
pharmacologique (anxiolytiques et autres prémédications), il existe une multitude d'approches psychologiques (programme de préparation psychologique, hypnose, musicothérapie, « clown-thérapie », ...). Enfin, la présence des parents lors de l'induction anesthésique est une approche innovante à l'origine de controverses entre les professionnels.

\subsubsection{Approches pharmacologiques}

Il existe de nombreuses molécules qui peuvent être utilisées comme prémédication a visée anxiolytique. Je limiterai mon propos à la plus courante d'entres elles, le midazolam. Cette molécule, de la famille des benzodiazépines, est commercialisée en France sous le nom d'Hypnovel $^{\circledR}$. Elle a notamment des propriétés anxiolytiques, amnésiantes, hypnotiques, anticonvulsives, myorelaxantes et sédatives, qui la rendent particulièrement utile en anesthésie. Il est établi que le midazolam permet de réduire nettement l'anxiété préopératoire chez l'enfant et l'adulte. Cependant, cette molécule n'est pas sans inconvénient chez l'enfant ; elle augmenterait la durée d'hospitalisation, la fréquence des troubles comportementaux postopératoires et l'amnésie [42].

\subsubsection{Approches psychologiques}

Les programmes de préparation à l'hospitalisation ont donné lieu à de nombreuses recherches depuis une trentaine d'années, en particulier dans les pays anglo-saxons. Ils permettent en effet de diminuer l'anxiété préopératoire tant chez l'adulte [8,9] que chez l'enfant [10,12]. Ces programmes s'appuient généralement sur le modèle des thérapies comportementales et cognitives. Selon les cas, il peut s'agir de techniques de psychoéducation, de restructuration cognitive, ou d'apprentissage de la relaxation et/ou de l'hypnose. Le contenu doit être adapté à l'âge des participants et à leur expérience préalable de l'hospitalisation. On peut retenir que l'objectif général est de favoriser le développement de stratégies pour faire face à l'anxiété préopératoire. Ces moyens psychologiques sont de réels atouts, tant pour les patients que pour les équipes, mais ils nécessitent un personnel disponible et formé. En outre, la grande diversité des approches rend difficiles les comparaisons des résultats entre les différents programmes.

D'autres approches psychologiques plus spécifiques ont donné lieu à des résultats intéressants. Dans une étude chez 52 adultes hospitalisés pour chirurgie, l'hypnose permettait de diminuer le score d'anxiété préopératoire à l'induction, alors qu'il augmentait pour les 
26 sujets contrôles $(-56 \%$ vs. $+47 \%, \mathrm{p}<0.001$ ) [33]. Chez l'enfant, une étude sur 50 sujets a montré que le nombre d'enfants anxieux était moins important dans le groupe hypnose (39\% vs $68 \%$, p <0.05) [2]. Toujours dans cette étude, les enfants du groupe hypnose avaient deux fois moins de risque d'avoir des scores importants de troubles comportementaux postopératoires un jour et une semaine après l'intervention. La musicothérapie permettrait elle aussi de diminuer l'anxiété préopératoire, mais les résultats des études sont contradictoires [29]. Chez l'enfant, deux intéressantes études randomisées et contrôlées ont évalué la « clown-thérapie ». La présence de clowns en préopératoire s'est révélée être efficace sur le score d'anxiété dans les deux cas : $\mathrm{n}=40$, score m-YPAS $=30.75$ vs. 50, $\mathrm{p}<0.05$ [40], $\mathrm{n}=55$, score $\mathrm{m}-\mathrm{YPAS}=42$ vs 50, $\mathrm{p}<0.05[7]$.

\subsubsection{La présence des parents}

Chez l'enfant, la présence des parents à l'induction fait l'objet de vives controverses [22]. Plusieurs enquêtes effectuées aux États-Unis ont établi que la majorité des parents désirent être présents et pensent pouvoir être utiles [11]. De nombreux travaux ont été menés pour déterminer les avantages et les inconvénients liés à cette pratique [42]. En 2005, Piira et al. ont examiné 13 études qui évaluaient l'impact de la présence des parents sur l'anxiété préopératoire chez l'enfant [31]. Seules les études où les parents n'avaient pas été assignés systématiquement ou de façon aléatoire dans le groupe «présent à l'induction » ou «absent à l'induction » ont obtenu des résultats positifs concernant l'anxiété préopératoire. Il semblerait donc que certains parents peuvent être plus «aidants » que d'autres. Kain et al. ont ainsi montré que la présence d'un parent calme à l'induction diminue l'anxiété de son enfant. À l'inverse, la présence d'un parent anxieux à l'induction n'a pas d'impact positif sur l'anxiété de son enfant [13]. Dans l'article précédemment cité, Piira suggère que les parents présents à l'induction pourraient bénéficier de conseils sur ce qu'ils peuvent faire ou non. Cela permettrait de potentialiser l'impact positif de la présence des parents à l'induction.

\section{Conclusion}

L'anxiété préopératoire est un phénomène courant, lié à l'appréhension d'une intervention chirurgicale qui peut entraîner des complications médicales et psychologiques. Il 
est établi que des niveaux importants d'anxiété préopératoire augmentent le risque de complications postopératoires chez l'enfant comme chez l'adulte.

L'absence d'échelles d'évaluations validées sur une population française rend difficile la prise en compte de ce trouble par les professionnels de santé. Il existe pourtant des moyens pharmacologiques et psychologiques qui permettent de diminuer l'anxiété préopératoire de manière significative. En préalable à toute intervention, il est cependant indispensable de disposer de moyens fiables d'identification des patients à risque.

Les professionnels impliqués dans le champ périopératoire, et notamment les psychologues cliniciens et les psychiatres, ont un important rôle à jouer du fait de leurs compétences en matière d'évaluation, de prévention, et de prise en charge psychothérapeutique des conséquences liées à l'anxiété préopératoire.

\section{Conflit d'intérêt : aucun.}

\section{Références}

[1] Beydon L, Dima CE. Anxiété périopératoire : évaluation et prévention. Le praticien en anesthésie réanimation 2007;13:161-70.

[2] Calipel S, Lucas-Polomeni MM, Wodey E, Ecoffey C. Premedication in children: hypnosis versus midazolam. Paediatr Anaesth 2005;15:275-81.

[3] Caumo W, Schmidt AP, Schneider CN, Bergmann J, Iwamoto CW, Adamatti LC, et al. Risk factors for postoperative anxiety in adults. Anaesthesia 2001;56:720-8.

[4] Cohen-Salmon D. En travers de la gorge. Paris: InterEditions; 1994.

[5] Denollet J. Emotional distress and fatigue in coronary heart disease: the Global Mood Scale (GMS). Psychol Med 1993;23:111-21.

[6] Freud A, Bergman T. Les enfants malades. Paris: Privat; 1976.

[7] Golan G, Tighe P, Dobija N, Perel A, Keidan I. Clowns for the prevention of preoperative anxiety in children: a randomized controlled trial. Paediatr Anaesth 2009;19:262-6.

[8] Horne DJ, Vatmanidis P, Careri A. Preparing patients for invasive medical and surgical procedures. 2: Using psychological interventions with adults and children. Behav Med 1994;20:15-21.

[9] Horne DJ, Vatmanidis P, Careri A. Preparing patients for invasive medical and surgical procedures. 1: Adding behavioral and cognitive interventions. Behav Med 1994;20:5-13. 
[10] Kain ZN, Caldwell-Andrews AA. Preoperative psychological preparation of the child for surgery: an update. Anesthesiol Clin North America 2005;23:597-614.

[11] Kain ZN, Caldwell-Andrews AA, Wang SM, Krivutza DM, Weinberg ME, Mayes LC. Parental intervention choices for children undergoing repeated surgeries. Anesth Analg 2003;96:970-5.

[12] Kain ZN, Caramico LA, Mayes LC, Genevro JL, Bornstein MH, Hofstadter MB. Preoperative preparation programs in children: a comparative examination. Anesth Analg 1998;87:1249-55.

[13] Kain ZN, Mayes LC, Caldwell-Andrews AA, Saadat H, McClain B, Wang SM. Predicting which children benefit most from parental presence during induction of anesthesia. Paediatr Anaesth 2006;16:627-34.

[14] Kain ZN, Mayes LC, Cicchetti DV, Bagnall AL, Finley JD, Hofstadter MB. The Yale Preoperative Anxiety Scale: how does it compare with a "gold standard"? Anesth Analg 1997;85:783-8.

[15] Kain ZN, Mayes LC, O'Connor TZ, Cicchetti DV. Preoperative anxiety in children. Predictors and outcomes. Arch Pediatr Adolesc Med 1996;150:1238-45.

[16] Kain ZN, Mayes LC, Wang SM, Caramico LA, Krivutza DM, Hofstadter MB. Parental presence and a sedative premedicant for children undergoing surgery: a hierarchical study. Anesthesiology 2000;92:939-46.

[17] Kain ZN, Mayes LC, Weisman SJ, Hofstadter MB. Social adaptability, cognitive abilities, and other predictors for children's reactions to surgery. J Clin Anesth 2000;12:54954.

[18] Kain ZN, Sevarino F, Pincus S, Alexander GM, Wang SM, Ayoub C, et al. Attenuation of the preoperative stress response with midazolam: effects on postoperative outcomes. Anesthesiology 2000;93:141-7.

[19] Kain ZN, Wang SM, Mayes LC, Caramico LA, Hofstadter MB. Distress during the induction of anesthesia and postoperative behavioral outcomes. Anesth Analg 1999;88:10427.

[20] Kindler CH, Harms C, Amsler F, Ihde-Scholl T, Scheidegger D. The visual analog scale allows effective measurement of preoperative anxiety and detection of patients' anesthetic concerns. Anesth Analg 2000;90:706-12.

[21] Mackenzie JW. Daycase anaesthesia and anxiety. A study of anxiety profiles amongst patients attending a day bed unit. Anaesthesia 1989;44:437-40. 
[22] McCann ME, Kain ZN. The management of preoperative anxiety in children: an update. Anesth Analg 2001;93:98-105.

[23] McGraw T. Preparing children for the operating room: psychological issues. Can J Anaesth 1994;41:1094-103.

[24] Melamed BG, Siegel LJ. Reduction of anxiety in children facing hospitalization and surgery by use of filmed modeling. J Consult Clin Psychol 1975;43:511-21.

[25] Miller KM, Wysocki T, Cassady JF, Jr., Cancel D, Izenberg N. Validation of measures of parents' preoperative anxiety and anesthesia knowledge. Anesth Analg 1999;88:251-7.

[26] Miller SM. Coping with impending stress: psychophysiological and cognitive correlates of choice. Psychophysiology 1979;16:572-81.

[27] Miller SM. Monitoring and blunting: validation of a questionnaire to assess styles of information seeking under threat. J Pers Soc Psychol 1987;52:345-53.

[28] Miller SM, Mangan CE. Interacting effects of information and coping style in adapting to gynecologic stress: should the doctor tell all? J Pers Soc Psychol 1983;45:223-36.

[29] Nilsson U. The anxiety- and pain- reducing effects of music interventions: a systematic review. AORN J 2008;87:780-807.

[30] Oberle K, Wry J, Paul P, Grace M. Environment, anxiety, and postoperative pain. West J Nurs Res 1990;12:745-53.

[31] Piira T, Sugiura T, Champion GD, Donnelly N, Cole AS. The role of parental presence in the context of children's medical procedures: a systematic review. Child Care Health Dev 2005;31:233-43.

[32] Roberston J, Freud A. A Mother's Observations on the Tonsillectomy of her Four-YearOld Daughter. Psychoanalytic Study of the Child 1956;11:410-33.

[33] Saadat H, Drummond-Lewis J, Maranets I, Kaplan D, Saadat A, Wang SM, et al. Hypnosis reduces preoperative anxiety in adult patients. Anesth Analg 2006;102:1394-6.

[34] Seeman RG, Rockoff MA. Preoperative anxiety: the pediatric patient. Int Anesthesiol Clin 1986;24:1-15.

[35] Shevde K, Panagopoulos G. A survey of 800 patients' knowledge, attitudes, and concerns regarding anesthesia. Anesth Analg 1991;73:190-8.

[36] Sipowicz RR, Vernon DT. Psychologial responses of children to hospitalization. A compaison of hospitalized and nonhospitalized twins. Am J Dis Child 1965;109:228-31.

[37] Spielberger CD. The state-trait anxiety inventory for children. Palo Alto; 1973.

[38] Spielberger CD, Gorsuch RL, Lushene RE. State Trait Anxiety Inventory for adults manual. Palo Alto, CA: Consulting Psychologists Press; 1983. 
[39] Thompson RH. Information seeking coping and anxiety in school-age children anticipating surgery. J Child Health Care 1994;23:87-97.

[40] Vagnoli L, Caprilli S, Robiglio A, Messeri A. Clown doctors as a treatment for preoperative anxiety in children: a randomized, prospective study. Pediatrics 2005;116:e563e567.

[41] Vetter TR. The epidemiology and selective identification of children at risk for preoperative anxiety reactions. Anesth Analg 1993;77:96-9.

[42] Watson AT, Visram A. Children's preoperative anxiety and postoperative behaviour. Paediatr Anaesth 2003;13:188-204.

[43] Wright KD, Stewart SH, Finley GA, Buffett-Jerrott SE. Prevention and intervention strategies to alleviate preoperative anxiety in children: a critical review. Behav Modif 2007;31:52-79.

[44] Yip P, Middleton P, Cyna AM, Carlyle AV. Non-pharmacological interventions for assisting the induction of anaesthesia in children. Cochrane Database Syst Rev 2009;CD006447.

\section{Annexe 1 : m-YPAS (modified Yale Preoperative Anxiety Scale) [14]}

Cette échelle d'évaluation a été développée pour évaluer l'anxiété préopératoire au moment de l'attente avant l'intervention et de l'induction de l'anesthésie. La m-YPAS comprend 22 items répartis en cinq catégories (activité, comportement verbal, expression, éveil, attitude avec les parents). Dans toutes les catégories, on retient l'item correspondant au niveau d'anxiété de l'enfant. Compte tenu du fait que les catégories ont un nombre d'items différents (quatre ou six), des quotients sont calculés puis additionnés afin d'obtenir un score total allant de zéro à cent.

Par exemple, pour un protocole avec un score de un pour les cinq catégories, le calcul est : $(1 / 4+1 / 6+1 / 4+1 / 4+1 / 4) \times 100 / 5=$ score total. Les enfants ayant un score inférieur ou égal à 24 sont considérés comme calmes, et ceux dont le score est supérieur à 24 comme anxieux. Elle a été traduite, mais n'a pas été validée en français. 


\begin{tabular}{|c|c|c|c|}
\hline \multirow{5}{*}{ Activité } & & cotation & réponse \\
\hline & $\begin{array}{l}\text { Curiosité, explore l'environnement, comportement adapté à l'âge } \\
\text { (jeux) }\end{array}$ & 1 & \\
\hline & $\begin{array}{l}\text { Ne joue pas, tripote ou suce ses doigts } \\
\text { Attente près de ses parents }\end{array}$ & 2 & \\
\hline & $\begin{array}{l}\text { Activité confuse sans but } \\
\text { Agrippé à ses parents } \\
\text { Bouge sur la table, refuse le masque }\end{array}$ & 3 & \\
\hline & $\begin{array}{l}\text { Refuse la séparation avec ses parents } \\
\text { Fuite, repousse les personnes à l'aide de ses membres }\end{array}$ & 4 & \\
\hline \multirow[t]{6}{*}{$\begin{array}{l}\text { Comportement } \\
\text { Verbal }\end{array}$} & $\begin{array}{l}\text { Questionne calmement, rires } \\
\text { Commentaires adaptés }\end{array}$ & 1 & \\
\hline & $\begin{array}{l}\text { Réponse chuchotée, parle comme un «bébé » } \\
\text { Hochement de tête }\end{array}$ & 2 & \\
\hline & Muet, pas de réponse aux adultes & 3 & 8 \\
\hline & Plaintes, gémissements, «pleurnichard » & 4 & \\
\hline & Cris, dit «non » & 5 & \\
\hline & Cris forts, audibles sous le masque & 6 & \\
\hline \multirow[t]{4}{*}{ Expression } & Heureux, souriant & 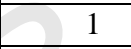 & \\
\hline & Indifférent, sans expression & 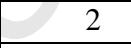 & \\
\hline & Inquiet, triste, yeux apeurés & 3 & \\
\hline & Anxieux, cris, effrayé & 4 & \\
\hline \multirow[t]{4}{*}{ Eveil } & Curiosité, en éveil & 1 & \\
\hline & $\begin{array}{l}\text { Repli, suce son pouce } \\
\text { Assis sans bouger, regarde vers les adultes }\end{array}$ & 2 & \\
\hline & $\begin{array}{l}\text { Vigilant, tendu } \\
\text { Regards furtifs }\end{array}$ & 3 & \\
\hline & $\begin{array}{l}\text { Paniqué, cris } \\
\text { Repousse les adultes }\end{array}$ & 4 & \\
\hline \multirow{4}{*}{$\begin{array}{l}\text { Attitude } \\
\text { Avec parents }\end{array}$} & Jeux, comportement adapté, pas besoin d'eux & 1 & \\
\hline & Recherche le confort, la sécurité, s'appuie contre eux & 2 & \\
\hline & Epie leurs gestes, s'accroche à eux & 3 & \\
\hline & $\begin{array}{l}\text { Rejet des parents ou désespérément accroché à eux } \\
\text { Ne les laissant pas s'éloigner }\end{array}$ & 4 & \\
\hline
\end{tabular}




\section{Annexe 2 : APAIS (Amsterdam preoperative anxiety and information scale) [25]}

Chaque item se cote de un à cinq selon l'intensité de l'adhésion à la phrase. Les items 1, 2, 4, 5 cotent l'anxiété. Pour obtenir le score d'anxiété, on additionne ces quatre items. Les sujets sont considérés comme anxieux lorsqu'ils ont un score supérieur à 11 pour ces quatre items. Les items trois et six cotent le désir d'information. Pour obtenir le score de désir d'information, on additionne ces deux items. Un score de deux à quatre traduirait un « refus d'information », un score entre cinq et sept un «désir moyen d'information », et un score supérieur à sept un «désir avide d'information ». Elle a été traduite par Beydon et Dima [1], mais n'a pas été validée en français.

\begin{tabular}{|c|c|c|c|c|c|c|}
\hline \multicolumn{2}{|r|}{ Item } & 1 & 2 & 3 & 4 & 5 \\
\hline 1 & Je suis préoccupé(e) par l'anesthésie & & & & & \\
\hline 2 & Je pense continuellement à l'anesthésie & & & & & \\
\hline 3 & J'aimerais en savoir le plus possible sur l'anesthésie & & & & & \\
\hline 4 & je suis préoccupé(e) par l'intervention & & & & & \\
\hline 5 & Je pense continuellement à l'intervention & & & & & \\
\hline 6 & Je voudrais en savoir le plus possible sur l'intervention & & & & & \\
\hline
\end{tabular}

